\begin{tabular}{|c|l|}
\hline Title & On self-similar solutions to the surface diffusion flow equations with contact angle boundary conditions \\
\hline Author(s) & A sai, Tomoro; GIGA, Y OSHIKAZU \\
\hline Citation & Hokkaido University Preprint Series in Mathematics, 1039, 1-25 \\
\hline Issue Date & 2013-9-17 \\
\hline DOI & 10.14943/84183 \\
\hline Doc URL & http://hdl.handle.net/2115/69843 \\
\hline Type & bulletin (article) \\
\hline File Information & pre1039.pdf \\
\hline
\end{tabular}

Instructions for use 


\title{
On self-similar solutions to the surface diffusion flow equations with contact angle boundary conditions
}

\author{
Tomoro Asai \\ Department of Mathematics, Hokkaido University, \\ Kita 10, Nishi 8, Kita-Ku, Sapporo, Hokkaido, 060-0810, JAPAN \\ (atomoro@math.sci.hokudai.ac.jp) \\ Yoshikazu Giga \\ Graduate School of Mathematical Sciences \\ University of Tokyo, Komaba 3-8-1, Meguro-ku, Tokyo 153-8914, JAPAN \\ (labgiga@ms.u-tokyo.ac.jp)
}

September 10, 2013

\begin{abstract}
We consider the surface diffusion flow equation when the curve is given as the graph of a function $v(x, t)$ defined in a half line $\mathbf{R}^{+}=\{x>0\}$ under the boundary conditions $v_{x}=$ $\tan \beta>0$ and $v_{x x x}=0$ at $x=0$. We construct a unique (spatially bounded) self-similar solution when the angle $\beta$ is sufficiently small. We further prove the stability of this self-similar solution. The problem stems from an equation proposed by W. W. Mullins (1957) to model formation of surface grooves on the grain boundaries, where the second boundary condition $v_{x x x}=0$ is replaced by zero slope condition on the curvature of the graph.

For construction of a self-similar solution we solves the initial-boundary problem with homogeneous initial data. However, since the problem is quasilinear and initial data is not compatible with the boundary condition a simple application of an abstract theory for quasilinear parabolic equation is not enough for our purpose. We use a semi-divergence structure to construct a solution.
\end{abstract}

2010 Mathematics Subject Classification: Primary 35C06; Secondary 35G31, 35K59, 74N20. Keywords: Self-similar solution; Surface diffusion flow; Stability; Analytic semigroup; Mild solution.

\section{Introduction}

We consider the initial-boundary problem for the surface diffusion flow equation of the form

$$
\frac{\partial v}{\partial t}=-\frac{\partial}{\partial x}\left[\frac{1}{\left(1+v_{x}^{2}\right)^{1 / 2}} \frac{\partial}{\partial x}\left(\frac{v_{x x}}{\left(1+v_{x}^{2}\right)^{3 / 2}}\right)\right], \quad x>0, t>0,
$$




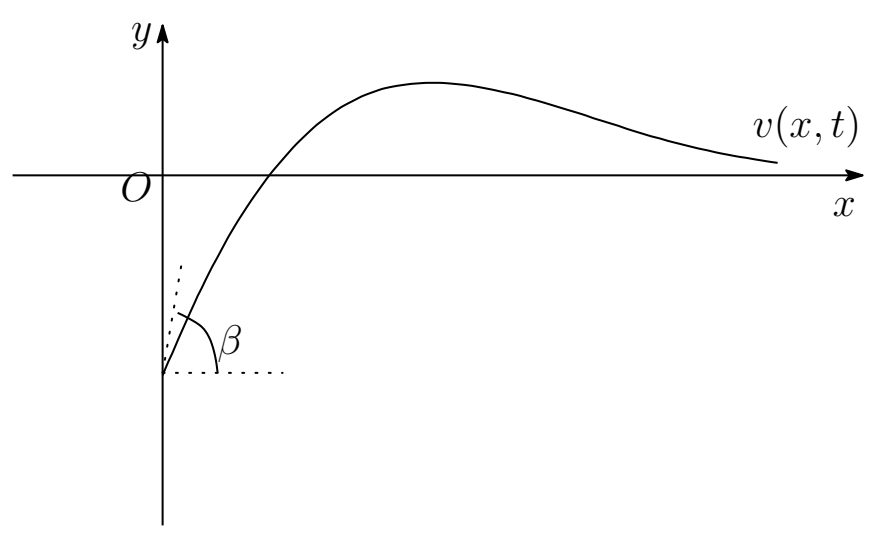

Figure 1: Profile of thermal groove

with the boundary condition

$$
\begin{array}{r}
v_{x}=\tan \beta, \quad x=0, t>0, \\
v_{x x x}=0, \quad x=0, t>0,
\end{array}
$$

and the initial condition

$$
v=a, \quad x>0, t=0,
$$

where $\beta$ is a nonnegative number and $v_{x}=\partial v / \partial x, v_{x x x}=\partial^{3} v / \partial x^{3}$.

We are interested in finding a solution for small $\beta>0$ and small bounded $a$. In particular, we shall find a bounded self-similar solution and discuss its stability. We say that a solution of (1.1)(1.3) is self-similar if the rescaled function

$$
v_{\lambda}(x, t)=\frac{1}{\lambda} v\left(\lambda x, \lambda t^{4}\right)
$$

satisfies $v_{\lambda}(x, t)=v(x, t)$ for all $\lambda>0$. By definition, a self-similar solution $v$ is of the form $v(x, t)=t^{1 / 4} Z\left(x / t^{1 / 4}\right)$ with some function of one variable $Z$ called a profile function. Evidently, $(\tan \beta) x$ is a trivial self-similar solution but is unbounded. Note that a self-similar solution corresponds a solution of (1.1)-(1.3) with zero initial data. In this paper we prove that the system (1.1)(1.4) is solvable globally-in-time and it asymptotically converges to a self-similar solution for large time provided that $a$ and $\beta$ are small.

This problem stems from a model describing the development of the surface groove proposed by W. W. Mullins [21]. There the condition (1.3) is replaced by no-flux condition $\kappa_{s}=0$ where $\kappa$ is the upward curvature of the graph curve $y=v(x, t)$ and $s$ is the arc-length parameter. Moreover, $a$ is assumed to be zero so that the initial surface is flat.

The equation (1.1) is the surface diffusion law $V=-\kappa_{s s}$ for the graph curve $y=v(x, t)$ where $V$ is the upward normal velocity. The condition (1.2) says that the contact angle of the curve $y=v(x, t)$ at the wall equals $\pi / 2-\beta$. 
Mullins [21] linearized the equation (1.1) and the boundary condition (1.3) around $v=0$ and studied the linear problem of the form

$$
\frac{\partial y}{\partial t}=-\frac{\partial^{4} y}{\partial x^{4}}, \quad x>0, t>0
$$

with the boundary condition

$$
\begin{array}{r}
y_{x}=\tan \beta, \quad x=0, t>0, \\
y_{x x x}=0, \quad x=0, t>0,
\end{array}
$$

and the initial condition

$$
y=0, \quad x>0, t=0 .
$$

The solution is again expected to be self-similar. Mullins applied the Laplace transform and derived the depth $y(0, t)$ which is proportional to $t^{1 / 4}$. Then he studied a profile function $Z$ solving the ordinary differential equation of the form

$$
Z^{\prime \prime \prime \prime}-\frac{1}{4} z Z^{\prime}+\frac{1}{4} Z=0
$$

Mullins assumed $Z$ to be a power series $Z=\sum_{n=0}^{\infty} a_{n} z^{n}$. He showed that $\left\{a_{n}\right\}$ can be determined by a recursion relation. However, its convergence was not proved.

P. A. Martin [20] improves and extends the results of Mullins. He studies the same problem (1.5)(1.8). However, the technique developed by Martin is different. He uses the Fourier cosine transform with respect to $x$. By this technique, he obtains the explicit formula for the solution $y$. He also considers the behavior of the solution at space infinity. In the latter half of [20], he studies multi-groove systems such as periodic surface profile case and two grooves case.

Note that for the original Mullins' system (1.1)-(1.2) with $\kappa_{s}=0$, it is not known whether or not bounded self-similar solutions exist. In this paper we linearized the boundary condition $\kappa_{s}=0$ to get (1.3) to prove the existence of a self-similar solution. Since (1.1) is quasilinear, such a result was not known even for our simplified problem.

There are two approaches to construct a self-similar solution. One is to solve an ordinary differential equation (ODE) for a profile function. For our problem this seem to be difficult since one has to solve a nonlinear equation of order 4 globally for $x>0$. Another way is a partial differential equation (PDE) approach initiated by Giga and Miyakawa [14] and developed by Cazenave and Weissler [6]. The main idea is to solve (1.1)-(1.3) by imposing a homogeneous initial data (in our case we consider zero initial data). One advantage of PDE method over ODE is that it is easy to show the stability of a constructed self-similar solution.

Although there is a large literature for solvability of the surface diffusion equation (e.g., [2, 3, $9,10])$, there are a few papers discussing the boundary value problem (e.g., $[16,17,11,12,13]$ ). Moreover, in our setting we have to handle initial data like $a=0$ which is incompatible with the boundary condition. We first transform the problem with homogeneous boundary condition by 
subtracting a solution $y=U^{L}$ of the linearized problem (1.5)-(1.8). To solve $u=v-U^{L}$ we rearrange the equation

$$
\partial_{t} u=-\partial_{x}^{4} u-\partial_{x} \Xi\left(u_{x}+U_{x}^{L},\left(u+U^{L}\right)_{x x},\left(u+U^{L}\right)_{x x x}\right), \quad x>0, t>0,
$$

with the boundary condition

$$
\begin{aligned}
u_{x}=0, & x=0, t>0, \\
u_{x x x}=0, & x=0, t>0 .
\end{aligned}
$$

The highest order term in $\Xi$ is linear in $\left(u+U^{L}\right)_{x x x}$ and its coefficient equals $\left(1+\left(u_{x}+U_{x}^{L}\right)^{2}\right)^{-2}-1$ which is very small when $u_{x}$ and $\beta$ are close to zero (so that $U_{x}^{L}$ is also close to zero). We solve this equation in $B U C_{1-\alpha}\left(J, h_{\text {even }}^{3+\gamma}(\mathbf{R})\right) \cap L^{\infty}\left(J, B U C_{\text {even }}^{1}(\mathbf{R})\right)$ (see Section 2 for definition) by adjusting an abstract method of Da Prato-Grisvard [8] and Angenent [1]. The smallness condition is invoked so that contraction mapping principle works. Note that $L^{p}$ type space is not suitable in handling this problem since we seek homogeneous functions.

Recently, Hamamuki [15] studies the self-similar solutions to the evaporation-condensation problem which is of the form

$$
\frac{\partial_{t} w}{\left(1+w_{x}^{2}\right)^{1 / 2}}=1-e^{-\kappa}, \quad x>0, t>0
$$

with the boundary condition

$$
\frac{\partial w}{\partial x}=\tan \beta, \quad x=0, t>0
$$

This problem (1.12)-(1.13) was also proposed as an evaporation-condensation model by Mullins [21]. The equation (1.12) is, of course, nonlinear. However, since the equation is second order, he is able to apply the viscosity solution theory to study the problem (1.12)-(1.13). He obtains the self-similar solution in a viscosity sense without the assumption that the angle $\beta>0$ is sufficiently small. His approach is quite different from the approach we discuss in this paper. His technique seems not to be applicable to our problem (1.1)-(1.3) since our problem is fourth order.

We next state several works related to our study. Broadbridge and Tritscher [5] search for a integrable and linearizable form of the equation (1.1) subject to boundary conditions. By transforming the variables, they reduces the equation to a linear ODE. Then, they solve the linear ODE and obtain an exact solution.

Kanel, Novick-Cohen and Vilenkin [18] prove the existence and uniqueness for a travelling wave solution for a problem in which motion by surface diffusion is coupled with mean curvature. By employing an angle formulation and a scaled arc-length parametrization, they reduce the problem to the solution of a third order ODE. They treat this problem via integral formulations using the Green functions.

Zhu [22] studies the existence of the stationary solution to the equation (1.1) with zero boundary conditions in the open interval $\Omega=(a, b) \subset \mathbf{R}$. His technique is to estimate for the free energy function which he introduces in [22]. 
This paper is organized as follows. In Section 2, we study the linearized equation and cite the result of P. A. Martin. We also give the definitions of some function spaces and show that the biLaplacian operator $-\partial_{x}^{4}$ generates the non $C_{0}$-bounded analytic semigroup on $L_{\mathrm{even}}^{\infty}$. In Section 3, we construct the mild solution of the problem (1.1)-(1.4). Finally, we prove the stability of self-similar solution.

A self-similar solution is constructed in a similar way in [4] but for the differential form of (1.1). The spatial derivative of the self-similar solution we construct in this paper is actually the solution of [4]. However, in [4] it is not clear the self-similar solution in [4] is bounded. Also, stability of the self-similar solution is not discussed in [4].

\section{Linear equation with boundary conditions}

\subsection{Explicit formula for the linear problem}

In this subsection, we study the linear problem (1.5)-(1.8). In order to solve the linear problem (1.5)(1.8), we recall the result of the paper by P. A. Martin [20] (Martin denoted $\tan \beta$ as $\theta_{\mathrm{eq}}$ ).

Lemma 2.1. There is an explicit formula of the solution for the problem (1.5)-(1.8)

$$
U^{L}(x, t)=-\frac{2 \tan \beta}{\pi} \int_{0}^{\infty}\left(1-e^{-k^{4} t}\right) \frac{\cos k x}{k^{2}} d k,
$$

which decays exponentially as $x \rightarrow \infty$.

Proof. See [20].

\subsection{Function spaces}

In this paper, we consider our problem on the half line $\mathbf{R}^{+}$. However, in the sequel, we extend the solution as an even function on the whole line $\mathbf{R}$. This extension as an even function is natural because our problem is the half side of triple junction for the surface diffusion flow. Thus, we shall use the function spaces of even functions. Now we introduce some Banach spaces, which shall be used later.

Definition 2.2. For $k=1,2, \ldots, 0<\nu<1, L_{\text {even }}^{\infty}(\mathbf{R}), B U C_{\text {even }}^{k}(\mathbf{R})$ and $C_{\text {even }}^{k+\nu}(\mathbf{R})$ are defined by

$$
\begin{aligned}
L_{\text {even }}^{\infty}(\mathbf{R}) & :=\left\{\varphi \in L^{\infty}(\mathbf{R}) ; \varphi \text { is even }\right\}, \\
B U C_{\text {even }}^{k}(\mathbf{R}) & :=\left\{\varphi \in B U C^{k}(\mathbf{R}) ; \varphi^{(l)} \in B U C(\mathbf{R})(l=0, \ldots, k) \text { and } \varphi \text { is even }\right\}, \\
B U C_{\text {even }}^{k+\nu}(\mathbf{R}) & :=\left\{\varphi \in B U C_{\text {even }}^{k}(\mathbf{R}) ; \varphi^{(k)} \in B U C^{\nu}(\mathbf{R})\right\} .
\end{aligned}
$$

$h^{k+\nu}(\mathbf{R})$ denotes the little Hölder space and it is identified with

$$
h^{k+\nu}(\mathbf{R}):=\left\{\varphi \in B U C^{k+\nu}(\mathbf{R}) ; \lim _{\delta \rightarrow 0} \sup _{\substack{x \neq y \\|x-y|<\delta}} \frac{\left|D^{k} \varphi(x)-D^{k} \varphi(y)\right|}{|x-y|^{\nu}}=0\right\},
$$


and $h_{\text {even }}^{k+\nu}(\mathbf{R})$ is defined by

$$
h_{\text {even }}^{k+\nu}(\mathbf{R}):=\left\{\varphi \in h^{k+\nu}(\mathbf{R}) ; \varphi \text { is even }\right\}
$$

Definition 2.3. For $T>0$ set $J=[0, T], \dot{J}=J \backslash\{0\}$. Let $0<\mu<1$ be fixed.

$$
B U C_{1-\mu}(J, E):=\left\{u \in C(\dot{J}, E) ;\left[t \mapsto t^{1-\mu} u\right] \in B U C(\dot{J}, E), \lim _{t \rightarrow 0+} t^{1-\mu}\|u(t)\|_{E}=0\right\}
$$

where $E$ is a (real or complex) Banach space.

\subsection{Analytic semigroup generated by the bi-Laplace operator}

In this subsection we shall give a proof that the bi-Laplace operator $-\partial_{x}^{4}$ generates non $C_{0}$-bounded analytic semigroup in $L^{\infty}$ type spaces. Such a result is essentially, known; see e.g., [19]. However, we give a proof for completeness and the reader's convenience.

Let us consider the resolvent equation

$$
\left(\lambda+\partial_{x}^{4}\right) u=f, \quad \text { for } \lambda \in \mathbb{C} \backslash\{0\},|\arg \lambda|<\pi
$$

in a formal way. We take the Fourier transform of the both sides to get

$$
\hat{u}(\xi)=\left(\lambda+|\xi|^{4}\right)^{-1} \hat{f}(\xi)
$$

(This calculation is justified when $u$ and $f$ are Schwartz' tempered distributions). Applying the inverse Fourier transformation one obtains

$$
u(x)=K^{\lambda} * f(x)
$$

with

$$
K^{\lambda}(x):=\frac{1}{\sqrt{2 \pi}} \int_{-\infty}^{\infty} \frac{e^{i x \xi}}{\lambda+\xi^{4}} d \xi . \quad(i=\sqrt{-1})
$$

Lemma 2.4. For given $\theta_{0} \in(0, \pi)$, there is a constant $C_{\theta_{0}}$ such that

$$
\left\|K^{\lambda} * f\right\|_{\infty} \leq C_{\theta_{0}}|\lambda|^{-1}\|f\|_{\infty}
$$

for all $f \in L^{\infty}(\mathbf{R}), \lambda \in \Sigma_{\theta_{0}}:=\left\{\lambda \in \mathbb{C} \backslash\{0\} ;|\arg \lambda| \leq \theta_{0}\right\}$.

Proof. To calculate (2.1), we first calculate

$$
K_{\theta}(x):=\frac{1}{\sqrt{2 \pi}} \int_{-\infty}^{\infty} \frac{e^{i x \xi}}{e^{i \theta}+\xi^{4}} d \xi, \quad \text { for } 0<\theta \leq \theta_{0}<\pi .
$$

Put

$$
\zeta=\exp (i \theta / 4), \quad \omega=\exp (i \pi / 4)
$$


Then the roots of $e^{i \theta}+\xi^{4}$ are $\zeta \omega, i \zeta \omega, i^{2} \zeta \omega$ and $i^{3} \zeta \omega$. Hereafter, we denote the residue of $f$ at the point $a$ by $\operatorname{Res}(f, a)$. By residue theorem, we have

$$
\begin{aligned}
& \int_{-\infty}^{\infty} \frac{e^{i x \xi}}{e^{i \theta}+\xi^{4}} d \xi \\
& = \begin{cases}2 \pi i\left[\operatorname{Res}\left(\frac{e^{i x \xi}}{e^{i \theta}+\xi^{4}}, \zeta \omega\right)+\operatorname{Res}\left(\frac{e^{i x \xi}}{e^{i \theta}+\xi^{4}}, i \zeta \omega\right)\right], & \text { if } x>0, \\
-2 \pi i\left[\operatorname{Res}\left(\frac{e^{i x \xi}}{e^{i \theta}+\xi^{4}}, i^{2} \zeta \omega\right)+\operatorname{Res}\left(\frac{e^{i x \xi}}{e^{i \theta}+\xi^{4}}, i^{3} \zeta \omega\right)\right], & \text { if } x<0 .\end{cases}
\end{aligned}
$$

We calculate the residues in (2.4) respectively.

$$
\begin{aligned}
\operatorname{Res}\left(\frac{e^{i x \xi}}{e^{i \theta}+\xi^{4}}, \zeta \omega\right) & =\frac{e^{i \zeta \omega x}}{(\zeta \omega-i \zeta \omega)\left(\zeta \omega-i^{2} \zeta \omega\right)\left(\zeta \omega-i^{3} \zeta \omega\right)} \\
& =\frac{\exp (-\zeta x / \sqrt{2}) \cdot \exp (i \zeta x / \sqrt{2})}{4 \zeta^{3} \omega^{3}} \\
\operatorname{Res}\left(\frac{e^{i x \xi}}{e^{i \theta}+\xi^{4}}, i \zeta \omega\right) & =-\frac{\exp (-\zeta x / \sqrt{2}) \cdot \exp (-i \zeta x / \sqrt{2})}{4 i \zeta^{3} \omega^{3}} \\
\operatorname{Res}\left(\frac{e^{i x \xi}}{e^{i \theta}+\xi^{4}}, i^{2} \zeta \omega\right) & =-\frac{\exp (\zeta x / \sqrt{2}) \cdot \exp (-i \zeta x / \sqrt{2})}{4 \zeta^{3} \omega^{3}} \\
\operatorname{Res}\left(\frac{e^{i x \xi}}{e^{i \theta}+\xi^{4}}, i \zeta \omega\right) & =\frac{\exp (\zeta x / \sqrt{2}) \cdot \exp (i \zeta x / \sqrt{2})}{4 i \zeta^{3} \omega^{3}}
\end{aligned}
$$

Thus from (2.4)-(2.6), we have when $x>0$

$$
\int_{-\infty}^{\infty} \frac{e^{i x \xi}}{e^{i \theta}+\xi^{4}} d \xi=\frac{\pi}{\sqrt{2} \zeta^{3}}\left(\sin \frac{\zeta x}{\sqrt{2}}+\cos \frac{\zeta x}{\sqrt{2}}\right) \exp \left(-\frac{\zeta x}{\sqrt{2}}\right)
$$

Similarly, from (2.4), (2.7) and (2.8), we have when $x<0$

$$
\int_{-\infty}^{\infty} \frac{e^{i x \xi}}{e^{i \theta}+\xi^{4}} d \xi=\frac{\pi}{\sqrt{2} \zeta^{3}}\left(-\sin \frac{\zeta x}{\sqrt{2}}+\cos \frac{\zeta x}{\sqrt{2}}\right) \exp \left(\frac{\zeta x}{\sqrt{2}}\right) .
$$

From (2.9) and (2.10) we can conclude that

$$
\int_{-\infty}^{\infty} \frac{e^{i x \xi}}{e^{i \theta}+\xi^{4}} d \xi=\frac{\pi}{\sqrt{2} \zeta^{3}}\left(\sin \frac{\zeta|x|}{\sqrt{2}}+\cos \frac{\zeta|x|}{\sqrt{2}}\right) \exp \left(-\frac{\zeta|x|}{\sqrt{2}}\right)
$$


To show that $K_{\theta}(x)$ is integrable for $0<\theta \leq \theta_{0}<\pi$, we first compute

$$
\begin{aligned}
\left(\sin \frac{\zeta|x|}{\sqrt{2}}\right) \exp \left(-\frac{\zeta|x|}{\sqrt{2}}\right) & \exp \left(\frac{i \zeta|x|}{\sqrt{2}}\right)-\exp \left(\frac{-i \zeta|x|}{\sqrt{2}}\right) \\
= & \frac{1 i}{2 i} \exp \left(-\frac{\zeta|x|}{\sqrt{2}}\right) \\
= & \frac{1}{2 i}\left[\exp \left(\frac{(-1+i) \zeta|x|}{\sqrt{2}}\right)-\exp \left(\frac{(-1-i) \zeta|x|}{\sqrt{2}}\right)\right] \\
= & \frac{1}{2 i}\left[\exp \left(-\frac{|x|}{\sqrt{2}}\left(\cos \frac{\theta}{4}+\sin \frac{\theta}{4}\right)\right) \cdot \exp \left(\frac{i|x|}{\sqrt{2}}\left(\cos \frac{\theta}{4}-\sin \frac{\theta}{4}\right)\right)\right. \\
& \left.-\exp \left(-\frac{|x|}{\sqrt{2}}\left(\cos \frac{\theta}{4}-\sin \frac{\theta}{4}\right)\right) \cdot \exp \left(-\frac{i|x|}{\sqrt{2}}\left(\cos \frac{\theta}{4}+\sin \frac{\theta}{4}\right)\right)\right], \\
( & \left.\cos \frac{\zeta|x|}{\sqrt{2}}\right) \exp \left(-\frac{\zeta|x|}{\sqrt{2}}\right) \\
= & \frac{\exp \left(\frac{i \zeta|x|}{\sqrt{2}}\right)+\exp \left(\frac{-i \zeta|x|}{\sqrt{2}}\right)}{2} \cdot \exp \left(-\frac{\zeta|x|}{\sqrt{2}}\right) \\
= & \frac{1}{2}\left[\exp \left(\frac{(-1+i) \zeta|x|}{\sqrt{2}}\right)+\exp \left(\frac{(-1-i) \zeta|x|}{\sqrt{2}}\right)\right] \\
= & \frac{1}{2}\left[\exp \left(-\frac{|x|}{\sqrt{2}}\left(\cos \frac{\theta}{4}+\sin \frac{\theta}{4}\right)\right) \cdot \exp \left(\frac{i|x|}{\sqrt{2}}\left(\cos \frac{\theta}{4}-\sin \frac{\theta}{4}\right)\right)\right. \\
& \left.-\exp \left(-\frac{|x|}{\sqrt{2}}\left(\cos \frac{\theta}{4}-\sin \frac{\theta}{4}\right)\right) \cdot \exp \left(-\frac{i|x|}{\sqrt{2}}\left(\cos \frac{\theta}{4}+\sin \frac{\theta}{4}\right)\right)\right] .
\end{aligned}
$$

Note that $\cos (\theta / 4)-\sin (\theta / 4)>0$ because of $0<\theta<\pi$. Thus, from (2.11)-(2.13)

$$
\int_{\mathbf{R}}\left|K_{\theta}(x)\right| d x \leq \frac{\pi}{2} \int_{\mathbf{R}}\left[\exp \left(-\frac{|x|}{\sqrt{2}}\left(\cos \frac{\theta}{4}+\sin \frac{\theta}{4}\right)\right)+\exp \left(-\frac{|x|}{\sqrt{2}}\left(\cos \frac{\theta}{4}-\sin \frac{\theta}{4}\right)\right)\right] d x .
$$

Since the right hand side of (2.3) is bounded by a constant $C_{\theta_{0}}$ for $\theta \in\left(0, \theta_{0}\right]$ with $\theta_{0} \in(0, \pi)$, we observe that

$$
\left\|K_{\theta}\right\|_{L^{1}(\mathbf{R})} \leq C_{\theta_{0}} \quad \text { for } \theta \in\left(0, \theta_{0}\right] .
$$

Take $\lambda \in \Sigma_{\theta_{0}}$, then by changing the variable $\xi=r^{1 / 4} \eta$ and recalling the definition of $K^{\lambda}(x)$ in (2.1), we have

$$
\begin{aligned}
K^{\lambda}(x) & =\frac{1}{\sqrt{2 \pi}} \int_{-\infty}^{\infty} \frac{e^{i x \xi}}{\lambda+\xi^{4}} d \xi \\
& =\frac{1}{\sqrt{2 \pi}} \int_{-\infty}^{\infty} \frac{e^{i x \xi}}{r e^{i \theta}+\xi^{4}} d \xi \\
& =\frac{1}{\sqrt{2 \pi}} \frac{1}{r^{3 / 4}} \int_{-\infty}^{\infty} \frac{e^{i r^{1 / 4} x \eta}}{e^{i \theta}+\eta^{4}} d \eta=\frac{1}{r^{3 / 4}} K_{\theta}\left(r^{1 / 4} x\right) .
\end{aligned}
$$


Thus from (2.15), we have

$$
\begin{aligned}
\left\|K^{\lambda}\right\|_{L^{1}} & =\int_{-\infty}^{\infty}\left|K^{\lambda}(x)\right| d x=\int_{-\infty}^{\infty} \frac{1}{r^{3 / 4}}\left|K_{\theta}\left(r^{1 / 4} x\right)\right| d x \\
& =\frac{1}{r} \int_{-\infty}^{\infty}\left|K_{\theta}(y)\right| d y=\frac{1}{r}\left\|K_{\theta}\right\|_{L^{1}} .
\end{aligned}
$$

From (2.14), (2.16) and Young's inequality, we have

$$
\begin{aligned}
\left\|\left(K^{\lambda} * f\right)\right\|_{L^{\infty}} & \leq\left\|K^{\lambda}\right\|_{L^{1}} \cdot\|f\|_{L^{\infty}} \\
& \leq C_{\theta_{0}}|\lambda|^{-1}\|f\|_{L^{\infty}}, \quad \text { for } \lambda \in \Sigma_{\theta_{0}} .
\end{aligned}
$$

We set

$$
\left(K^{\lambda} f\right)(x):=\left(K^{\lambda} * f\right)(x)
$$

for $f \in L^{\infty}(\mathbf{R})$. We define a closed linear operator $A$ (formally corresponding to $-\partial_{x}^{4}$ ) in $L^{\infty}(\mathbf{R})$ such that $\lambda+A$ is the inverse operator of $K^{\lambda}$. The definition is independent of a choice of $\lambda$ because of a resolvent identity. The domain $D(A)$ of $A$ equals the range of $K^{\lambda}$ so that $D(A)=$ $W^{4, \infty}(\mathbf{R})$ which is not dense in $L^{\infty}(\mathbf{R})$. Note that $K^{\lambda}$ preserve $L_{\text {even }}^{\infty}(\mathbf{R})$ one can define $A$ in $L_{\text {even }}^{\infty}(\mathbf{R})$. Lemma 2.4 implies that $-A$ generates a non $C_{0}$-bounded analytic semigroup in $L^{\infty}(\mathbf{R})$ and $L_{\text {even }}^{\infty}(\mathbf{R})$. If one restricts the space in $B U C(\mathbf{R})$, then $D(A)=B U C^{4}(\mathbf{R})$, so $A$ is densely defined.

Theorem 2.5. The operator $A\left(=-\partial_{x}^{4}\right)$ is the infinitesimal generator of a (non $C_{0^{-}}$) bounded analytic semigroup in $L^{\infty}(\mathbf{R})$ and $L_{\text {even }}^{\infty}(\mathbf{R})$. The semigroup is an bounded $C_{0}$-analytic semigroup if one restricts the space into $B U C(\mathbf{R})$ and $B U C_{\text {even }}(\mathbf{R})$ respectively.

\section{Quasilinear equation with linear boundary conditions}

In this Section, we study our problem (1.1)-(1.4).

\subsection{Hölder estimate for the perturbed term}

Hereafter, we shall estimate the term $\Xi\left(v_{x}, v_{x x}, v_{x x x}\right)=h\left(v_{x}\right) \partial_{x}^{3} v-g\left(v_{x}, v_{x x}\right)$ with Hölder seminorm $h^{\gamma}$, where

$$
h(r)=\frac{1}{\left(1+r^{2}\right)^{2}}-1, \quad g(p, q)=\frac{3 p q^{2}}{\left(1+p^{2}\right)^{3}} .
$$

Thus, we give preliminary lemmas to estimate $\Xi$.

Lemma 3.1. Let $F$ be a function of $C^{1}$-class. Then

$$
\begin{aligned}
{[\varphi \psi]_{h^{\gamma}} } & \leq|\varphi|_{\infty}[\psi]_{h^{\gamma}}+[\varphi]_{h^{\gamma}}|\psi|_{\infty}, \\
{[F(\varphi)]_{h^{\gamma}} } & \leq \sup \left|F^{\prime}\right| \cdot[\varphi]_{h^{\gamma}} \quad \text { for all } \varphi, \psi \in h^{\gamma}(\mathbf{R}) .
\end{aligned}
$$


Proof. We just recall the definition of Hölder semi-norm. Then we have

$$
\begin{aligned}
{[\varphi \psi]_{h^{\gamma}} } & =\sup _{x, y} \frac{|\varphi(x) \psi(x)-\varphi(y) \psi(y)|}{|x-y|^{\gamma}} \\
& =\sup _{x, y} \frac{|\varphi(x)(\psi(x)-\psi(y))+\psi(y)(\varphi(x)-\varphi(y))|}{|x-y|^{\gamma}} \\
& \leq \sup _{x, y}|\varphi(x)| \frac{|\psi(x)-\psi(y)|}{|x-y|^{\gamma}}+\sup _{x, y}|\psi(y)| \frac{|\varphi(x)-\varphi(y)|}{|x-y|^{\gamma}} \\
& \leq|\varphi|_{\infty}[\psi]_{h^{\gamma}}+[\varphi]_{h^{\gamma}}|\psi|_{\infty} .
\end{aligned}
$$

Similarly, we have

$$
\begin{aligned}
{[F(\varphi)]_{h^{\gamma}} } & =\sup _{x, y} \frac{|F(\varphi(x))-F(\varphi(y))|}{|x-y|^{\gamma}} \\
& =\sup _{x, y} \frac{\int_{0}^{1}\left|\frac{d}{d \theta} F(\theta \varphi(x)+(1-\theta) \varphi(y)) d \theta\right|}{|x-y|^{\gamma}} \\
& =\sup _{x, y} \frac{\left|\left(\int_{0}^{1} F^{\prime}(\theta \varphi(x)+(1-\theta) \varphi(y)) d \theta\right) \cdot(\varphi(x)-\varphi(y))\right|}{|x-y|^{\gamma}} \\
& \leq \sup _{x, y}\left(\int_{0}^{1}\left|F^{\prime}(\theta \varphi(x)+(1-\theta) \varphi(y))\right| d \theta\right) \frac{|\varphi(x)-\varphi(y)|}{|x-y|^{\gamma}} \\
& \leq \sup _{\mid}\left|F^{\prime}\right| \cdot[\varphi]_{h^{\gamma}} .
\end{aligned}
$$

Lemma 3.2. There are constants $\widetilde{C}_{1}$ and $\widehat{C}_{1}$ such that

$$
\begin{gathered}
{\left[h\left(v_{x}\right) v_{x x x}\right]_{h^{\gamma}} \leq \widetilde{C}_{1}\left\{\left|v_{x}\right|_{\infty}\left[v_{x x x}\right]_{h^{\gamma}}+\left[v_{x}\right]_{h^{\gamma}}\left|v_{x x x}\right|_{\infty}\right\}} \\
{\left[g\left(v_{x}, v_{x x}\right)\right]_{h^{\gamma}} \leq \widehat{C}_{1}\left\{\left|v_{x}\right|_{\infty}\left[v_{x x}^{2}\right]_{h^{\gamma}}+\left[v_{x}\right]_{h^{\gamma}}\left|v_{x x}^{2}\right|_{\infty}\right\} .}
\end{gathered}
$$

holds for all $v \in h^{3+\gamma}(\mathbf{R})$.

Proof. By Lemma 3.1, we have

$$
\begin{aligned}
{\left[h\left(v_{x}\right) v_{x x x}\right]_{h^{\gamma}} } & \leq\left|h\left(v_{x}\right)\right|_{\infty}\left[v_{x x x}\right]_{h^{\gamma}}+\left[h\left(v_{x}\right)\right]_{h^{\gamma}}\left|v_{x x x}\right|_{\infty} \\
& \leq \sup \left|h^{\prime}\right| \cdot\left|v_{x}\right|_{\infty} \cdot\left[v_{x x x}\right]_{h^{\gamma}}+\sup \left|h^{\prime}\right| \cdot\left[v_{x}\right]_{h^{\gamma}} \cdot\left|v_{x x x}\right|_{\infty} \\
& \leq \widetilde{C}_{1}\left\{\left|v_{x}\right|_{\infty}\left[v_{x x x}\right]_{h^{\gamma}}+\left[v_{x}\right]_{h^{\gamma}}\left|v_{x x x}\right|_{\infty}\right\} .
\end{aligned}
$$

Similarly by setting $g_{1}\left(v_{x}\right):=3 v_{x} /\left(1+v_{x}^{2}\right)^{3}$, we have

$$
\begin{aligned}
{\left[g\left(v_{x}, v_{x x}\right)\right]_{h^{\gamma}} } & =\left[g_{1}\left(v_{x}\right) v_{x x}^{2}\right]_{h^{\gamma}} \\
& \leq\left|g_{1}\left(v_{x}\right)\right|_{\infty}\left[v_{x x}^{2}\right]_{h^{\gamma}}+\left[g_{1}\left(v_{x}\right)\right]_{h^{\gamma}}\left|v_{x x}^{2}\right|_{\infty} \\
& \leq \sup \left|g_{1}^{\prime}\right| \cdot\left|v_{x}\right|_{\infty} \cdot\left[v_{x x}^{2}\right]_{h^{\gamma}}+\sup \left|g_{1}^{\prime}\right| \cdot\left[v_{x}\right]_{h^{\gamma}} \cdot\left|v_{x x}^{2}\right|_{\infty} \\
& \leq \widehat{C}_{1}\left(\left|v_{x}\right|_{\infty}\left[v_{x x}^{2}\right]_{h^{\gamma}}+\left[v_{x}\right]_{h^{\gamma}}\left|v_{x x}^{2}\right|_{\infty}\right) .
\end{aligned}
$$




\subsection{Existence of mild solution}

Let us prove our main result of this paper.

Theorem 3.3. Let $\gamma$ be in $(0,1)$. Let $a(x) \in B U C_{\text {even }}^{1}(\mathbf{R})$ with $\|a\|_{B U C^{1}}$ sufficiently small. Then there exists $\beta_{0}>0$ such that if $\beta \in\left(0, \beta_{0}\right)$, there exists a unique mild solution (i.e., the integral solution $\left.u(t)=e^{-t \partial_{x}^{4}} a-\int_{0}^{t} e^{-(t-s) \partial_{x}^{4}} \partial_{x} \Xi\left(u_{x}+U_{x}^{L}, u_{x x}+U_{x x}^{L}, u_{x x x}+U_{x x x}^{L}\right)(s) d s\right)$ of (1.9)-(1.11) which satisfies

$$
u \in B U C_{1-\alpha}\left(J, h_{\text {even }}^{3+\gamma}(\mathbf{R})\right) \cap L^{\infty}\left(J, B U C_{\text {even }}^{1}(\mathbf{R})\right),
$$

where $\alpha=1 / 2-\gamma / 4$ and $J=[0, T]$ for any $T>0$.

Proof. We first prove that the initial data equals zero. We set

$$
F_{0}:=L_{\text {even }}^{\infty}(\mathbf{R}), \quad F_{1}:=B U C_{\text {even }}^{4}(\mathbf{R}), \quad A:=\partial_{x}^{4} .
$$

By Theorem 2.5, $-A$ is the infinitesimal generator of a bounded analytic semigroup on $F_{0}$. We remark that

$$
h_{\text {even }}^{\gamma}(\mathbf{R})=\left(F_{0}, F_{1}\right)_{\gamma / 4, \infty}^{0}, \quad h_{\text {even }}^{3+\gamma}(\mathbf{R})=\left(F_{0}, F_{1}\right)_{(3+\gamma) / 4, \infty}^{0},
$$

for the definition of a continuous interpolation space, see [7] and [19]. We also set

$$
\theta:=\gamma / 4, \quad F_{\theta}:=\left(F_{0}, F_{1}\right)_{\theta, \infty}^{0},
$$

thus $h_{\text {even }}^{\gamma}(\mathbf{R})=F_{\theta}$. Next, for positive constants $M, M_{\infty}>0$, we set

$$
\begin{aligned}
& \mathcal{Z}_{M, M_{\infty}}(J):=\left\{u \in B U C_{1-\alpha}\left(J, h_{\mathrm{even}}^{3+\gamma}(\mathbf{R})\right) \cap L^{\infty}\left(J, B U C_{\mathrm{even}}^{1}(\mathbf{R})\right)\right. \\
&\left.u(0)=0,\|u\|_{B U C_{1-\alpha}\left(J, h_{\mathrm{even}}^{3+\gamma}(\mathbf{R})\right)} \leq M,\|u\|_{L^{\infty}\left(J, B U C_{\mathrm{even}}^{1}(\mathbf{R})\right)} \leq M_{\infty}\right\}
\end{aligned}
$$

equipped with the norm $\|u\|_{\mathcal{Z}_{M, M_{\infty}(J)}}:=\max \left(\|u\|_{B U C_{1-\alpha}\left(J, h_{\text {even }}^{3+\gamma}(\mathbf{R})\right)},\|u\|_{L^{\infty}\left(J, B U C_{\text {even }}^{1}(\mathbf{R})\right)}\right)$. We denote $m$ and $m_{\infty}$ by the norm of $\left\|U^{L}\right\|_{B U C_{1-\alpha}\left(J, h_{\text {even }}^{3+\gamma}(\mathbf{R})\right)}$ and $\left\|U^{L}\right\|_{L^{\infty}\left(J, B U C_{\text {even }}^{1}(\mathbf{R})\right)}$. From the explicit formula of $U^{L}$ in Lemma 2.1, if the contact angle $\beta>0$ is sufficiently small, then we can assume that $m$ and $m_{\infty}$ are sufficiently small. Note that the perturbed term $\Xi\left(v_{x}, v_{x x}, v_{x x x}\right)$ does not necessarily attain zero at $x=0$ because of the second order derivative $v_{x x}$. For this reason, we introduce "the odd functionalized operator" $\mathcal{P}$ as

$$
\mathcal{P}: h^{\gamma}\left(\mathbf{R}_{+}\right) \rightarrow h_{\text {odd }}^{\gamma}(\mathbf{R}) ; \varphi \mapsto \begin{cases}\varphi(x)-\varphi(0), & \text { if } x \geq 0 \\ -(\varphi(-x)-\varphi(0)), & \text { if } x<0\end{cases}
$$

Then for $u \in \mathcal{Z}_{M, M_{\infty}}(J)$, we consider the integral solution

$$
\Gamma u:=-\int_{0}^{t} e^{-(t-s) A} \partial_{x}\left(\mathcal{P} \Xi\left(v_{x}, v_{x x}, v_{x x x}\right)\right)(s) d s .
$$

Note that $\partial_{x}(\mathcal{P} \Xi)$ is even because $\mathcal{P} \Xi$ is odd. 
We shall show that $\Gamma$ maps $\mathcal{Z}_{M, M_{\infty}}(J)$ into itself and has a fixed point in $\mathcal{Z}_{M, M_{\infty}}(J)$ which implies the existence of a unique solution of (1.9)-(1.11).

\section{Step 1 ( $\Gamma$ maps $\mathcal{Z}_{M, M_{\infty}}(J)$ into itself)}

For a fixed $T>0$, we introduce the equivalent norms for the little Hölder spaces $h^{\gamma}, h^{3+\gamma}$ and $B U C^{1}$ by

$$
\begin{aligned}
\|f\|_{h^{\gamma}(\mathbf{R})} & :=\frac{|f|_{\infty}}{T^{\theta}}+[f]_{h^{\gamma}(\mathbf{R})}, \\
\|f\|_{h^{3+\gamma}(\mathbf{R})} & :=\frac{|f|_{\infty}}{T^{3 / 4+\theta}}+\frac{\left|f^{\prime}\right|_{\infty}}{T^{1 / 2+\theta}}+\frac{\left|f^{\prime \prime}\right|_{\infty}}{T^{1 / 4+\theta}}+\frac{\left|f^{\prime \prime \prime}\right|_{\infty}}{T^{\theta}}+\left[f^{\prime \prime \prime \prime}\right]_{h \gamma(\mathbf{R})}, \\
\|f\|_{B U C^{1}(\mathbf{R})} & :=\frac{|f|_{\infty}}{T^{1 / 4}}+\left|f^{\prime}\right|_{\infty},
\end{aligned}
$$

where $|\cdot|$ is the $L^{\infty}$-norm and $[\cdot]$ is the little Hölder semi-norm. Hence we observe that

$$
\begin{aligned}
\|\Gamma u\|_{B U C_{1-\alpha}\left(J, h_{\text {even }}^{3+\gamma}(\mathbf{R})\right)}= & \sup _{t \in \dot{J}} t^{1-\alpha}\|\Gamma u\|_{h^{3+\gamma}(\mathbf{R})} \\
= & \frac{1}{T^{3 / 4+\theta}} \sup _{t \in \dot{J}} t^{1-\alpha}|\Gamma u|_{\infty}+\frac{1}{T^{1 / 2+\theta}} \sup _{t \in \dot{J}} t^{1-\alpha}\left|\partial_{x} \Gamma u\right|_{\infty} \\
& +\frac{1}{T^{1 / 4+\theta}} \sup _{t \in \dot{J}} t^{1-\alpha}\left|\partial_{x}^{2} \Gamma u\right|_{\infty}+\frac{1}{T^{\theta}} \sup _{t \in \dot{J}} t^{1-\alpha}\left|\partial_{x}^{3} \Gamma u\right|_{\infty} \\
& \quad+\sup _{t \in \dot{J}} t^{1-\alpha}\left[\partial_{x}^{3} \Gamma u\right]_{h^{\gamma}(\mathbf{R}) .}
\end{aligned}
$$

To estimate (3.6), we recall the definition of $F_{\theta}$ semi-norm, that is,

$$
[x]_{F_{\theta}}:=\sup _{0<r \leq 1} r^{1-\theta}\left\|A e^{-r A} x\right\|_{F_{0}} .
$$


First we shall calculate the last term of (3.6).

$$
\begin{aligned}
& t^{1-\alpha}\left[\partial_{x}^{3} \Gamma u\right]_{h^{\gamma}(\mathbf{R})} \\
& =t^{1-\alpha}\left[\int_{0}^{t} \partial_{x}^{3} e^{-(t-s) A} \partial_{x}\left(\mathcal{P} \Xi\left(v_{x}, v_{x x}, v_{x x x}\right)\right)(s) d s\right]_{h \gamma(\mathbf{R})} \\
& =t^{1-\alpha}\left[\int_{0}^{t} \partial_{x}^{4} e^{-(t-s) A} \mathcal{P} \Xi\left(v_{x}, v_{x x}, v_{x x x}\right)(s) d s\right]_{h \gamma(\mathbf{R})} \\
& \lesssim t^{1-\alpha}\left[\int_{0}^{t} A e^{-(t-s) A} \mathcal{P} \Xi\left(v_{x}, v_{x x}, v_{x x x}\right)(s) d s\right]_{F_{\theta}} \\
& \leq t^{1-\alpha}\left\{\left[\int_{0}^{t / 2} A e^{-(t-s) A} \mathcal{P} \Xi(s) d s\right]_{F_{\theta}}+\left[\int_{t / 2}^{t} A e^{-(t-s) A} \mathcal{P} \Xi(s) d s\right]_{F_{\theta}}\right\} \\
& \lesssim t^{1-\alpha}\left\{\int_{0}^{t / 2}\left\|A e^{-(t-s) A}\right\|_{\mathcal{L}\left(F_{\theta}, F_{\theta}\right)} \cdot[\mathcal{P} \Xi(s)]_{F_{\theta}} d s+\sup _{0<r \leq 1} r^{1-\theta}\left\|A_{t / 2}^{2} e^{-(r+t-s) A} \mathcal{P} \Xi(s)\right\|_{F_{\theta}} d s\right\} \\
& \lesssim t^{1-\alpha}\left\{\int_{0}^{t / 2}(t-s)^{-1}[\mathcal{P} \Xi(s)]_{F_{\theta}} d s\right. \\
& \left.+\sup _{0<r \leq 1} r^{1-\theta} \int_{t / 2}^{t}\left\|A^{2} e^{-(r+t-s) A}\right\|_{\mathcal{L}\left(F_{\theta}, F_{0}\right)} \cdot[\mathcal{P} \Xi(s)]_{F_{\theta}} d s\right\} \\
& \lesssim t^{1-\alpha}\left\{\int_{0}^{t / 2}(t-s)^{-1}[\mathcal{P} \Xi(s)]_{F_{\theta}} d s+\sup _{0<r \leq 1} r^{1-\theta} \int_{t / 2}^{t}(r+t-s)^{-2+\theta}[\mathcal{P} \Xi(s)]_{F_{\theta}} d s\right\} .
\end{aligned}
$$

Here we use the symbol $\lesssim$ when we suppress a numerical constant $C$ depending only on exponents. In other words, we simply write $a \lesssim b$ instead of $a \leq C b$.

To estimate $[\mathcal{P} \Xi(s)]_{h^{\gamma}(\mathbf{R})}=\left[h\left(v_{x}\right) \partial_{x}^{3} v-g\left(v_{x}, v_{x x}, v_{x x x}\right)\right]_{h^{\gamma}(\mathbf{R})}$, we shall use Lemma 3.2 and the interpolation inequality between $B U C^{1}$-norm and $h^{3+\gamma}$-norm as

$$
\begin{aligned}
{\left[\varphi^{\prime}\right]_{h^{\gamma}} } & \lesssim[\varphi]_{h^{1+\gamma}} \lesssim[\varphi]_{B U C^{1}}^{2 /(2+\gamma)}[\varphi]_{h^{3+\gamma}}^{\gamma /(2+\gamma)} \\
{\left[\left(\varphi^{\prime \prime}\right)^{2}\right]_{h^{\gamma}} } & \leq 2\left|\varphi^{\prime \prime}\right|_{\infty}\left[\varphi^{\prime \prime}\right]_{h^{\gamma}} \lesssim[\varphi]_{B U C^{2}} \cdot[\varphi]_{h^{2+\gamma}} \\
& \lesssim[\varphi]_{B U C^{1}}^{(1+\gamma) /(2+\gamma)}[\varphi]_{h^{3+\gamma}}^{1 /(2+\gamma)} \cdot[\varphi]_{B U C^{1}}^{1 /(2+\gamma)}[\varphi]_{h^{3+\gamma}}^{(1+\gamma) /(2+\gamma)} \\
& =c[\varphi]_{B U C^{1}}[\varphi]_{h^{3+\gamma}}
\end{aligned}
$$

Thus, we have

$$
\begin{aligned}
{\left[h\left(v_{x}\right) v_{x x x}\right]_{h^{\gamma}} } & \leq \widetilde{C}_{1}\left(\left|v_{x}\right|_{\infty}\left[v_{x x x}\right]_{h^{\gamma}}+\left[v_{x}\right]_{h^{\gamma}}\left|v_{x x x}\right|_{\infty}\right) \\
& \lesssim \widetilde{C}_{1}\left([v]_{B U C^{1}}[v]_{h^{3+\gamma}}+[v]_{B U C^{1}}^{2 /(2+\gamma)}[v]_{h^{3+\gamma}}^{\gamma /(2+\gamma)} \cdot[v]_{B U C^{1}}^{\gamma /(2+\gamma)}[v]_{h^{3+\gamma}}^{2 /(2+\gamma)}\right. \\
& \leq \widetilde{C}_{1}\left(\left(M_{\infty}+m_{\infty}\right)\left(s^{1-\alpha}[v]_{h^{3+\gamma}}\right) s^{\alpha-1}+[v]_{B U C^{1}}\left(s^{1-\alpha}[v]_{h^{3+\gamma}}\right) s^{\alpha-1}\right) \\
& \leq \widetilde{C}_{1}\left(\left(M_{\infty}+m_{\infty}\right)(M+m) s^{\alpha-1}+\left(M_{\infty}+m_{\infty}\right)(M+m) s^{\alpha-1}\right) \\
& =2 \widetilde{C}_{1}\left(M_{\infty}+m_{\infty}\right)(M+m) s^{\alpha-1}
\end{aligned}
$$




$$
\begin{aligned}
{\left[g\left(v_{x}, v_{x x}\right)\right]_{h^{\gamma}} } & \leq \widehat{C}_{1}\left\{\left|v_{x}\right|_{\infty}\left[v_{x x}^{2}\right]_{h^{\gamma}}+\left[v_{x}\right]_{h^{\gamma}}\left|v_{x x}\right|^{2}\right\} \\
& \lesssim \widehat{C}_{1}\left\{[v]_{B U C^{1}} \cdot[v]_{B U C^{1}}[v]_{h^{3+\gamma}}+[v]_{B U C^{1}}^{2 /(2+\gamma)}[v]_{h^{3+\gamma}}^{\gamma /(2+\gamma)} \cdot[v]_{B U C^{1}}^{2(1+\gamma) /(2+\gamma)}[v]_{h^{3+\gamma}}^{2 /(2+\gamma)}\right\} \\
& =\widehat{C}_{1}\left\{[v]_{B U C^{1}}^{2}[v]_{h^{3+\gamma}}+[v]_{B U C^{1}}^{2}[v]_{h^{3+\gamma}}\right\} \\
& =2 \widehat{C}_{1}\left([v]_{B U C^{1}}^{2}\left(s^{1-\alpha}[v]_{h^{3+\gamma}}\right) s^{\alpha-1}\right) \\
& =2 \widehat{C}_{1}\left(M_{\infty}+m_{\infty}\right)^{2}(M+m) s^{\alpha-1}
\end{aligned}
$$

Now we are in a position to estimate (3.7). We shall estimate the integrand

$$
r^{1-\theta} t^{1-\alpha} \int_{t / 2}^{t}(r+t-s)^{-2+\theta} s^{\alpha-1} d s
$$

by using Lemma A.1 in the Appendix as

$$
r^{1-\theta} t^{1-\alpha} \int_{t / 2}^{t}(r+t-s)^{-2+\theta} s^{\alpha-1} d s \leq \frac{2^{1-\alpha}}{1-\theta} .
$$

From (3.8)-(3.10), we have

$$
\begin{aligned}
& \lesssim t^{1-\alpha}\left\{\int_{0}^{t / 2}(t-s)^{-1}\left[\widetilde{C}_{1}\left(M_{\infty}+m_{\infty}\right)(M+m) s^{\alpha-1}+\widehat{C}_{1}\left(M_{\infty}+m_{\infty}\right)^{2}(M+m) s^{\alpha-1}\right] d s\right. \\
& \left.+\sup _{0<r \leq 1} r^{1-\theta} \int_{t / 2}^{t}(r+t-s)^{-2+\theta}\left[\widetilde{C}_{1}\left(M_{\infty}+m_{\infty}\right)(M+m) s^{\alpha-1}+\widehat{C}_{1}\left(M_{\infty}+m_{\infty}\right)^{2}(M+m) s^{\alpha-1} d s\right]\right\} \\
& \lesssim\left(\int_{0}^{1 / 2}(1-\sigma)^{-1} \sigma^{\alpha-1} d \sigma\right)\left[\widetilde{C}_{1}\left(M_{\infty}+m_{\infty}\right)(M+m)+\widehat{C}_{1}\left(M_{\infty}+m_{\infty}\right)^{2}(M+m)\right] \\
& +\frac{2^{1-\alpha}}{1-\theta}\left[\widetilde{C}_{1}\left(M_{\infty}+m_{\infty}\right)(M+m)+\widehat{C}_{1}\left(M_{\infty}+m_{\infty}\right)^{2}(M+m)\right] .
\end{aligned}
$$

Estimating for the other terms of (3.6) proceeds similarly. The difference is $L^{\infty}$-norm estimate for the integrand $t^{1-\alpha} \int_{0}^{t} \partial_{x}^{k} e^{-(t-s) A} \partial_{x}(\mathcal{P} \Xi) d s(k=0,1,2,3)$. For example, we estimate the fourth terms of (3.6) as

$$
\begin{aligned}
t^{1-\alpha}\left|\int_{0}^{t} \partial_{x}^{3} e^{-(t-s) A} \partial_{x}(\mathcal{P} \Xi) d s\right|_{\infty} & =t^{1-\alpha}\left|\int_{0}^{t} \partial_{x}^{4} e^{-(t-s) A} \mathcal{P} \Xi d s\right|_{\infty} \\
& \leq t^{1-\alpha} \int_{0}^{t}\left\|A e^{-(t-s) A} \mathcal{P} \Xi\right\|_{F_{0}} d s \\
& \leq t^{1-\alpha} \int_{0}^{t}\left\|A e^{-(t-s) A}\right\|_{\mathcal{L}\left(F_{\theta}, F_{0}\right)}[\mathcal{P} \Xi]_{F_{\theta}} d s \\
& \leq C t^{1-\alpha}\left(\int_{0}^{t}(t-s)^{-1+\theta}[\mathcal{P} \Xi]_{F_{\theta}} d s\right)
\end{aligned}
$$

Thus, estimating (3.12), the power $t^{\theta}$ shall appear, which cancels out the term $1 / T^{\theta}$ in (3.6). We also recall that from (3.5)

$$
\|\Gamma u\|_{L^{\infty}\left(J, B U C^{1}(\mathbf{R})\right)}=\frac{1}{T^{1 / 4}} \sup _{t \in \dot{J}}|\Gamma u|_{\infty}+\sup _{t \in \dot{J}}\left|\partial_{x} \Gamma u\right|_{\infty} .
$$


Thus, estimating (3.13) proceeds similarly as above. Since $m$ and $m_{\infty}$ are sufficiently small enough, then, with a suitable choices of $M$ and $M_{\infty}$, we can show that

$$
\max \left(\|\Gamma u\|_{B U C_{1-\alpha}\left(J, h_{\mathrm{even}}^{3+\gamma}(\mathbf{R})\right)},\|\Gamma u\|_{L^{\infty}\left(J, B U C_{\mathrm{even}}^{1}(\mathbf{R})\right)}\right) \leq \max \left(M, M_{\infty}\right)
$$

Thus, we have shown that $\Gamma\left(\mathcal{Z}_{M, M_{\infty}}(J)\right) \subset \mathcal{Z}_{M, M_{\infty}}(J)$.

Step 2 ( $\Gamma$ is a contraction on $\mathcal{Z}_{M, M_{\infty}}(J)$ ).

Pick $u_{i} \in \mathcal{Z}_{M, M_{\infty}}(J)(i=1,2)$. Let $v_{i}:=u_{i}+U^{L}(i=1,2)$ and

$$
\Xi_{i}\left(\left(v_{i}\right)_{x},\left(v_{i}\right)_{x x},\left(v_{i}\right)_{x x x}\right):=h\left(\left(v_{i}\right)_{x}\right)\left(v_{i}\right)_{x x x}-g\left(\left(v_{i}\right)_{x},\left(v_{i}\right)_{x x}\right)(i=1,2) .
$$

Then we have

$$
\Gamma u_{1}-\Gamma u_{2}=-\int_{0}^{t} e^{-(t-s) A} \partial_{x}\left(\mathcal{P}\left(\Xi_{1}-\Xi_{2}\right)\right)(s) d s
$$

where

$$
\begin{aligned}
\Xi_{1}-\Xi_{2}= & h\left(\left(v_{1}\right)_{x}\right)\left(v_{1}\right)_{x x x}-g\left(\left(v_{1}\right)_{x},\left(v_{1}\right)_{x x}\right) \\
& -h\left(\left(v_{2}\right)_{x}\right)\left(v_{2}\right)_{x x x}+g\left(\left(v_{2}\right)_{x},\left(v_{2}\right)_{x x}\right) \\
= & h\left(\left(v_{1}\right)_{x}\right)\left(u_{1}-u_{2}\right)_{x x x}+\left[h\left(\left(v_{1}\right)_{x}\right)-h\left(\left(v_{2}\right)_{x}\right)\right]\left(v_{2}\right)_{x x x} \\
& -\left[g\left(\left(v_{1}\right)_{x},\left(v_{1}\right)_{x x}\right)-g\left(\left(v_{2}\right)_{x},\left(v_{2}\right)_{x x}\right)\right] \\
= & : I_{1}+I_{2}+I_{3} .
\end{aligned}
$$

Now we estimate the right-hand side of (3.14) with respect to Hölder semi-norm respectively. The argument, however, proceeds similarly as in Step 1, therefore we leave the detailed computations to the reader.

$$
\begin{aligned}
{\left[I_{1}\right]_{h^{\gamma}} } & =\left[h\left(\left(v_{1}\right)_{x}\right)\left(u_{1}-u_{2}\right)_{x x x}\right]_{h^{\gamma}} \\
& \leq \widetilde{C}_{2}\left\{\left|\left(v_{1}\right)_{x}\right|_{\infty}\left[\left(u_{1}-u_{2}\right)_{x x x}\right]_{h^{\gamma}}+\left[\left(v_{1}\right)_{x}\right]_{h^{\gamma}}\left|\left(u_{1}-u_{2}\right)_{x x x}\right|_{\infty}\right\}
\end{aligned}
$$

Next, by the fundamental theorem of calculus, we observe that

$$
\begin{aligned}
I_{2} & =\left(h\left(\left(v_{1}\right)_{x}\right)-h\left(\left(v_{2}\right)_{x}\right)\right)\left(v_{2}\right)_{x x x} \\
& =\left(\int_{0}^{1} h^{\prime}\left(\theta\left(u_{1}\right)_{x}+(1-\theta)\left(u_{2}\right)_{x}+U_{x}^{L}\right) d \theta\right)\left(u_{1}-u_{2}\right)_{x}\left(v_{2}\right)_{x x x},
\end{aligned}
$$

where $h^{\prime}(v)=6 v /\left(1+v^{2}\right)^{4}$.

To estimate (3.16) with respect to Hölder semi-norm, we use Lemma 3.1 by

$$
\left[\varphi_{1} \varphi_{2} \varphi_{3}\right]_{h^{\gamma}} \leq\left[\varphi_{1}\right]_{h^{\gamma}}\left|\varphi_{2}\right|_{\infty}\left|\varphi_{3}\right|_{\infty}+\left|\varphi_{1}\right|_{\infty}\left[\varphi_{2}\right]_{h^{\gamma}}\left|\varphi_{3}\right|_{\infty}+\left|\varphi_{1}\right|_{\infty}\left|\varphi_{2}\right|_{\infty}\left[\varphi_{3}\right]_{h^{\gamma}}
$$


From (3.16) and (3.17), we have

$$
\begin{aligned}
{\left[I_{2}\right]_{h \gamma}=} & {\left[\left(h\left(\left(v_{1}\right)_{x}\right)-h\left(\left(v_{2}\right)_{x}\right)\right)\left(v_{2}\right)_{x x x}\right]_{h \gamma} } \\
\leq & \widetilde{C}_{3}\left\{\left(\int_{0}^{1}\left[h^{\prime}\left(\theta\left(u_{1}\right)_{x}+(1-\theta)\left(u_{2}\right)_{x}+U_{x}^{L}\right)\right]_{h^{\gamma}} d \theta\right)\left|\left(u_{1}-u_{2}\right)_{x}\right|_{\infty}\left|\left(v_{2}\right)_{x x x}\right|_{\infty}\right. \\
& +\left(\int_{0}^{1}\left|h^{\prime}\left(\theta\left(u_{1}\right)_{x}+(1-\theta)\left(u_{2}\right)_{x}+U_{x}^{L}\right)\right|_{\infty} d \theta\right)\left[\left(u_{1}-u_{2}\right)_{x}\right]_{h^{\gamma}}\left|\left(v_{2}\right)_{x x x}\right|_{\infty} \\
& \left.+\left(\int_{0}^{1}\left|h^{\prime}\left(\theta\left(u_{1}\right)_{x}+(1-\theta)\left(u_{2}\right)_{x}+U_{x}^{L}\right)\right|_{\infty} d \theta\right)\left|\left(u_{1}-u_{2}\right)_{x}\right|_{\infty}\left[\left(v_{2}\right)_{x x x}\right]_{h^{\gamma}}\right\} \\
\leq & \widetilde{C}_{3}\left\{\left(\left[u_{1}\right]_{h^{1+\gamma}}+\left[u_{2}\right]_{h^{1+\gamma}}+\left[U^{L}\right]_{h^{1+\gamma}}\right)\left[u_{1}-u_{2}\right]_{B U C^{1}}\left[v_{2}\right]_{B U C^{3}}\right. \\
+ & \left(\left[u_{1}\right]_{B U C^{1}}+\left[u_{2}\right]_{B U C^{1}}+\left[U^{L}\right]_{B U C^{1}}\right)\left[u_{1}-u_{2}\right]_{h^{1+\gamma}}\left|v_{2}\right|_{B U C^{3}} \\
+ & \left.\left(\left[u_{1}\right]_{B U C^{1}}+\left[u_{2}\right]_{B U C^{1}}+\left[U^{L}\right]_{B U C^{1}}\right)\left[u_{1}-u_{2}\right]_{B U C^{1}}\left[v_{2}\right]_{h^{3+\gamma}}\right\} .
\end{aligned}
$$

Estimating $\left[I_{3}\right]_{h^{\gamma}}$ is a similar argument. Thus, by the fundamental theorem of calculus, we observe that

$$
\begin{aligned}
g\left(p_{1}, q_{1}\right)-g\left(p_{2}, q_{2}\right)= & \left(\int_{0}^{1} D_{1} g\left(\theta p_{1}+(1-\theta) p_{2}, \theta q_{1}+(1-\theta) q_{2}\right) d \theta\right)\left(p_{1}-p_{2}\right) \\
& +\left(\int_{0}^{1} D_{2} g\left(\theta p_{1}+(1-\theta) p_{2}, \theta q_{1}+(1-\theta) q_{2}\right) d \theta\right)\left(q_{1}-q_{2}\right),
\end{aligned}
$$

where

$$
\begin{aligned}
D_{1} g & =\frac{3\left(1-5 p^{2}\right) q^{2}}{\left(1+p^{2}\right)^{4}}, & D_{2} g & =\frac{6 p q}{\left(1+p^{2}\right)^{3}}, \\
p_{i} & =\left(u_{i}+U^{L}\right)_{x}, & q_{i} & =\left(u_{i}+U^{L}\right)_{x x},(i=1,2) .
\end{aligned}
$$

We estimate the right-hand side of (3.19) with respect to Hölder semi-norm.

$$
\begin{aligned}
{\left[\left(D_{1} g\right)\left(p_{1}-p_{2}\right)\right]_{h^{\gamma}} \leq } & {\left[D_{1} g\right]_{h^{\gamma}}\left|p_{1}-p_{2}\right|_{\infty}+\left|D_{1} g\right|_{\infty}\left[p_{1}-p_{2}\right]_{h^{\gamma}} } \\
\leq & \left|\frac{3\left(1-5 p^{2}\right)}{\left(1+p^{2}\right)^{4}}\right|\left[q^{2}\right]_{h^{\gamma}}\left|p_{1}-p_{2}\right|_{\infty}+\left[\frac{3\left(1-5 p^{2}\right)}{\left(1+p^{2}\right)^{4}}\right]_{h^{\gamma}}\left|q^{2}\right|_{\infty}\left|p_{1}-p_{2}\right|_{\infty} \\
& +\left|\frac{3\left(1-5 p^{2}\right)}{\left(1+p^{2}\right)^{4}}\right|_{\infty}\left|q^{2}\right|_{\infty}\left[p_{1}-p_{2}\right]_{h^{\gamma}} \\
\leq & \widehat{C}_{2}\left\{|q|_{\infty}[q]_{h^{\gamma}}\left|p_{1}-p_{2}\right|_{\infty}+[p]_{h^{\gamma}}|q|_{\infty}^{2}\left|p_{1}-p_{2}\right|_{\infty}\right. \\
& \left.+|q|_{\infty}^{2}\left[p_{1}-p_{2}\right]_{h^{\gamma}}\right\}
\end{aligned}
$$




$$
\begin{aligned}
{\left[\left(D_{2} g\right)\left(q_{1}-q_{2}\right)\right]_{h^{\gamma}} \leq } & {\left[D_{2} g\right]_{h^{\gamma}}\left|q_{1}-q_{2}\right|_{\infty}+\left|D_{2} g\right|_{\infty}\left[q_{1}-q_{2}\right]_{h^{\gamma}} } \\
\leq & \left|\frac{6 p}{\left(1+p^{2}\right)^{3}}\right|_{\infty}[q]_{h^{\gamma}}\left|q_{1}-q_{2}\right|_{\infty}+\left[\frac{6 p}{\left(1+p^{2}\right)^{3}}\right]_{h^{\gamma}}|q|_{\infty}\left[q_{1}-q_{2}\right]_{h^{\gamma}} \\
& +\left|\frac{6 p}{\left(1+p^{2}\right)^{3}}\right|_{\infty}|q|_{\infty}\left[q_{1}-q_{2}\right]_{h^{\gamma}} \\
\leq & \widehat{C}_{3}\left\{[q]_{h^{\gamma}}\left|q_{1}-q_{2}\right|_{\infty}+[p]_{h \gamma}|q|_{\infty}\left[q_{1}-q_{2}\right]_{h^{\gamma}}\right. \\
& \left.+|q|_{\infty}\left[q_{1}-q_{2}\right]_{h^{\gamma}}\right\} .
\end{aligned}
$$

The estimates (3.20) and (3.21) yield that

$$
\begin{aligned}
& {\left[I_{3}\right]_{h \gamma} \leq \widehat{C}_{2}\left\{\left(\left|\left(u_{1}\right)_{x x}\right|_{\infty}\right.\right.}\left.+\left|\left(u_{2}\right)_{x x}\right|_{\infty}+\left|U_{x x}^{L}\right|_{\infty}\right) \\
& \times\left(\left[\left(u_{1}\right)_{x x}\right]_{h^{\gamma}}+\left[\left(u_{2}\right)_{x x}\right]_{h^{\gamma}}+\left[U_{x x}^{L}\right]_{h \gamma}\right)\left|u_{1}-u_{2}\right|_{\infty} \\
&+\left(\left[\left(u_{1}\right)_{x}\right]_{h^{\gamma}}+\left[\left(u_{2}\right)_{x}\right]_{h^{\gamma}}+\left[U_{x}^{L}\right]_{h \gamma}\right) \\
& \times\left(\left|\left(u_{1}\right)_{x x}\right|_{\infty}^{2}+\left|\left(u_{2}\right)_{x x}\right|_{\infty}^{2}+\left|U_{x x}^{L}\right|_{\infty}^{2}\right)\left|u_{1}-u_{2}\right|_{\infty} \\
&\left.\left(\left|\left(u_{1}\right)_{x x}\right|_{\infty}^{2}+\left|\left(u_{2}\right)_{x x}\right|_{\infty}^{2}+\left|U_{x x}^{L}\right|_{\infty}^{2}\right)\left[u_{1}-u_{2}\right]_{h^{\gamma}}\right\} \\
&+\widehat{C}_{3}\left\{\left(\left[\left(u_{1}\right)_{x x}\right]_{h^{\gamma}}+\left[\left(u_{2}\right)_{x x}\right]_{h \gamma}+\left[U_{x x}^{L}\right]_{h \gamma}\right)\left|\left(u_{1}\right)_{x x}-\left(u_{2}\right)_{x x}\right|_{\infty}\right. \\
&+\left(\left[\left(u_{1}\right)_{x}\right]_{h \gamma}+\left[\left(u_{2}\right)_{x}\right]_{h \gamma}+\left[U_{x}^{L}\right]_{h \gamma}\right)\left(\left|\left(u_{1}\right)_{x x}\right|_{\infty}+\left|\left(u_{2}\right)_{x x}\right|_{\infty}+\left|U_{x x}^{L}\right|_{\infty}\right) \\
& \times\left[\left(u_{1}\right)_{x x}-\left(u_{2}\right)_{x x}\right]_{h^{\gamma}} \\
&+\left(\left|\left(u_{1}\right)_{x x}\right|_{\infty}+\left|\left(u_{2}\right)_{x x}\right|_{\infty}+\left|U_{x x}^{L}\right|_{\infty}\right)\left[\left(u_{1}\right)_{x x}-\left(u_{2}\right)_{x x}\right]_{h \gamma} .
\end{aligned}
$$


From (3.15), (3.18) and (3.22), the term $\sup _{t \in j} t^{1-\alpha}\left[\partial_{x}^{3}\left(\Gamma u_{1}-\Gamma u_{2}\right)\right]_{h^{\gamma}}$ is estimated by

$$
\begin{aligned}
\sup _{t \in \dot{J}} t^{1-\alpha}\left[\partial_{x}^{3}\left(\Gamma u_{1}-\Gamma u_{2}\right)\right]_{h^{\gamma}} \leq\left(\int_{0}^{1 / 2}(1-\sigma)^{-1} \sigma^{\alpha-1} d \sigma+\frac{2^{1-\alpha}}{1-\theta}\right) \\
\times\left\{\widetilde{C}_{2}\left[\left(M_{\infty}+m_{\infty}\right)+\left(M_{\infty}+m_{\infty}\right)^{2 /(2+\gamma)}(M+m)^{\gamma /(2+\gamma)}\right]\right. \\
+\widetilde{C}_{3}\left[\left(2 M_{\infty}^{2 /(2+\gamma)} M^{\gamma /(2+\gamma)}+m_{\infty}^{2 /(2+\gamma)} m^{\gamma /(2+\gamma)}\right)\left(M_{\infty}+m_{\infty}\right)^{\gamma /(2+\gamma)}(M+m)^{2 /(2+\gamma)}\right. \\
\left.+\left(2 M_{\infty}+m_{\infty}\right)\left(M_{\infty}+m_{\infty}\right)^{\gamma /(2+\gamma)}(M+m)^{2 /(2+\gamma)}+\left(2 M_{\infty}+m_{\infty}\right)(M+m)\right] \\
+\widehat{C}_{2}\left[\left(2 M_{\infty}^{(1+\gamma) /(2+\gamma)} M^{1 /(2+\gamma)}+m_{\infty}^{(1+\gamma) /(2+\gamma)} m^{1 /(2+\gamma)}\right)\right. \\
\times\left(2 M_{\infty}^{1 /(2+\gamma)} M^{(1+\gamma) /(2+\gamma)}+m_{\infty}^{1 /(2+\gamma)} m^{(1+\gamma) /(2+\gamma)}\right) \\
\quad+\left(2 M_{\infty}^{2 /(2+\gamma)} M^{\gamma /(2+\gamma)}+m_{\infty}^{2 /(2+\gamma)} m^{\gamma /(2+\gamma)}\right) \\
\times\left(2 M_{\infty}^{2(1+\gamma) /(2+\gamma)} M^{2 /(2+\gamma)}+m_{\infty}^{2(1+\gamma) /(2+\gamma)} m^{2 /(2+\gamma)}\right) \\
\left.+\left(2 M_{\infty}^{2(1+\gamma) /(2+\gamma)} M^{2 /(2+\gamma)}+m_{\infty}^{2(1+\gamma) /(2+\gamma)} m^{1 /(2+\gamma)}\right)\right] \\
+\widehat{C}_{3}\left[\left(2 M_{\infty}^{1 /(2+\gamma)} M^{(1+\gamma) /(2+\gamma)}+m_{\infty}^{1 /(2+\gamma)} m^{(1+\gamma) /(2+\gamma)}\right)\right. \\
\quad+\left(2 M_{\infty}^{2 /(2+\gamma)} M^{\gamma /(2+\gamma)}+m_{\infty}^{2 /(2+\gamma)} m^{\gamma /(2+\gamma)}\right) \\
\times\left(2 M_{\infty}^{(1+\gamma) /(2+\gamma)} M^{1 /(2+\gamma)}+m_{\infty}^{(1+\gamma) /(2+\gamma)} m^{1 /(2+\gamma)}\right) \\
\left.\left.\quad+\left(2 M_{\infty}^{(1+\gamma) /(2+\gamma)} M^{1 /(2+\gamma)}+m_{\infty}^{(1+\gamma) /(2+\gamma)} m^{1 /(2+\gamma)}\right)\right]\right\}
\end{aligned}
$$

The estimates for the other terms

$$
\begin{aligned}
& \frac{1}{T^{\theta}} \sup _{t \in \dot{J}} t^{1-\alpha}\left|\partial_{x}^{3}\left(\Gamma u_{1}-\Gamma u_{2}\right)\right|_{\infty}, \quad \frac{1}{T^{1 / 4+\theta}} \sup _{t \in \dot{J}} t^{1-\alpha}\left|\partial_{x}^{2}\left(\Gamma u_{1}-\Gamma u_{2}\right)\right|_{\infty}, \\
& \frac{1}{T^{1 / 2+\theta}} \sup _{t \in \dot{J}} t^{1-\alpha}\left|\partial_{x}\left(\Gamma u_{1}-\Gamma u_{2}\right)\right|_{\infty}, \quad \frac{1}{T^{3 / 4+\theta}} \sup _{t \in \dot{J}} t^{1-\alpha}\left|\Gamma\left(u_{1}-u_{2}\right)\right|_{\infty}
\end{aligned}
$$

proceed similarly. Thus, we have actually estimated $\left\|\Gamma u_{1}-\Gamma u_{2}\right\|_{B U C_{1-\alpha}\left(J, h_{\mathrm{even}}^{3+\gamma}(\mathbf{R})\right)}$. The estimate of $\left\|\Gamma u_{1}-\Gamma u_{2}\right\|_{L^{\infty}\left(J, B U C_{\text {even }}^{1}(\mathbf{R})\right)}$ also proceed similarly as above.

The smallness of the constants $m, m_{\infty}$ and our suitable choices of the constants $M, M_{\infty}$ shows that $\left\|\Gamma u_{1}-\Gamma u_{2}\right\|_{\mathcal{Z}_{M, M_{\infty}}(J)}$ is less than or equal to $(1 / 2)\left\|u_{1}-u_{2}\right\|_{\mathcal{Z}_{M, M_{\infty}}(J)}$, which concludes that $\Gamma$ is a contraction on $\mathcal{Z}_{M, M_{\infty}}(J)$. By uniqueness the constructed solution is self-similar, i.e., $v_{\lambda}=v$.

The proof proceeds similarly in the case of $a \not \equiv 0$. What we have to do is to estimate $t^{1-\alpha}\left[\partial_{x}^{3} e^{-t A} a\right]_{h^{\gamma}}$.

$$
\begin{aligned}
t^{1-\alpha}\left[\partial_{x}^{3} e^{-t A} a\right]_{h^{\gamma}} & =t^{1-\alpha}\left[A^{1 / 2} e^{-t A} \partial_{x} a\right]_{h^{\gamma}} \\
& \leq t^{1-\alpha}\left\|A^{1 / 2} e^{-t A}\right\|_{\mathcal{L}\left(F_{0}, F_{\theta}\right)} \cdot\left\|a^{\prime}\right\|_{F_{0}} \\
& \leq t^{1-\alpha} \cdot t^{-1 / 2-\theta}\left\|a^{\prime}\right\|_{F_{0}} \\
& =\left\|a^{\prime}\right\|_{F_{0}} .
\end{aligned}
$$

In the last line of (3.24), we use the relation $\alpha=1 / 2-\gamma / 4$. Thus, if $\left\|a^{\prime}\right\|_{F_{0}}=\left|a^{\prime}\right|_{\infty}$ is sufficiently small, we can control the quantity $t^{1-\alpha}\left[\partial_{x}^{3} e^{-t A} a\right]_{h \gamma}$. Hence, we can show the unique existence of the mild solution with the initial data $a \not \equiv 0$. 
Remark. (i) It is likely that the constructed self-similar solution is smooth by using linear parabolic theory for higher order equation although we do not pursue this problem. Of course, the self-similar solution is a solution of ODE so it must be smooth.

(ii) The technique we used in the estimate (3.10) is similar to the technique of the proof of [1, Theorem 2.14] by Angenent which is the extension of Da Prato-Grisvard construction [8]. See the Appendix for details.

\subsection{Stability of self-similar solution}

In this subsection we discuss the stability of self-similar solution. Let $u$ be the mild solution which we constructed in Theorem 3.3. For $\lambda>0$, put

$$
u_{\lambda}(x, t):=\frac{1}{\lambda} u\left(\lambda x, \lambda^{4} t\right), \quad a_{\lambda}(x):=\frac{1}{\lambda} a(\lambda x) .
$$

Since $u$ is the mild solution of the problem (1.9)-(1.11), $u_{\lambda}$ is also the mild solution of the problem (1.9)-(1.11) with the initial data $a_{\lambda}$.

Theorem 3.4. The rescaled $u_{\lambda}$ uniformly converges to $\tilde{u}$ as $\lambda \rightarrow \infty$ on any compact sets in $(0, T] \times$ $\mathbf{R}$, where $\tilde{u}$ is the self-similar solution of (1.9)-(1.11). In particular, $t^{-1 / 4} u\left(t^{1 / 4} x, t\right) \rightarrow Z(x)$ as $t \rightarrow \infty$ when $Z$ is the profile function of $\tilde{u}$.

To show Theorem 3.4, we need a preliminary lemma. For the definition of the weighted Hölder space $C_{\beta}^{\alpha}((0, T], E)$, see [19, Chapter 4].

Lemma 3.5. Let $u$ be the mild solution which we constructed in Theorem 3.3. Then

$$
u \in C_{\frac{1+\gamma}{2}}^{\frac{1+\gamma}{4}}\left((0, T], h_{\text {even }}^{2+\gamma}(\mathbf{R})\right)
$$

and there exists $C=C\left(M, M_{\infty}\right)$ such that

$$
\|u\|_{C_{\frac{1+\gamma}{2}}^{\frac{1+\gamma}{4}}\left((0, T], h_{\text {even }}^{2+\gamma}(\mathbf{R})\right)} \leq C
$$

Proof.

$$
\begin{aligned}
u(t)-u(s) & =\left(e^{-t A}-e^{-s A}\right) a-\int_{s}^{t} e^{-(t-\tau) A} \partial_{x} \Xi(\tau) d \tau-\int_{0}^{s}\left(e^{-(t-\tau) A}-e^{-(s-\tau) A}\right) \partial_{x} \Xi(\tau) d \tau \\
& =-\int_{s}^{t} A e^{-r A} a d r-\int_{s}^{t} e^{-(t-\tau) A} \partial_{x} \Xi(\tau) d \tau+\int_{0}^{s} \int_{s-\tau}^{t-\tau} A e^{-r A} \partial_{x} \Xi(\tau) d r d \tau .
\end{aligned}
$$

Recalling the definition of the weighted Hölder space (cf. [19, Chapter 4]), we have

$$
[u]_{C_{\frac{1+\gamma}{2}}^{\frac{1+\gamma}{4}}\left(J, h_{\text {even }}^{2+\gamma}(\mathbf{R})\right)}=\sup _{0<\varepsilon<T} \varepsilon^{\frac{1+\gamma}{2}}[u]_{C^{\frac{1+\gamma}{4}}\left([\varepsilon, T], h_{\text {even }}^{2+\gamma}(\mathbf{R})\right)} .
$$


Thus, for $\varepsilon \leq s<t \leq T$ we calculate

$$
\begin{aligned}
\varepsilon^{\frac{1+\gamma}{2}}\left[\partial_{x}^{2} u(t)-\partial_{x}^{2} u(s)\right]_{h \gamma} \leq & \varepsilon^{\frac{1+\gamma}{2}}\left[-\partial_{x}^{2}\left(\int_{s}^{t} A e^{-r A} a d r\right)\right]_{h \gamma}+\varepsilon^{\frac{1+\gamma}{2}}\left[\partial_{x}^{2} \int_{s}^{t} e^{-(t-\tau) A} \partial_{x} \Xi(\tau) d \tau\right]_{h \gamma} \\
& +\varepsilon^{\frac{1+\gamma}{2}}\left[\partial_{x}^{2} \int_{0}^{s} \int_{s-\tau}^{t-\tau} A e^{-r A} \partial_{x} \Xi(\tau) d r d \tau\right]_{h^{\gamma}} \\
= & : J_{1}+J_{2}+J_{3} .
\end{aligned}
$$

$J_{1}$ is estimated by

$$
\begin{aligned}
J_{1} & \leq \varepsilon^{\frac{1+\gamma}{2}}\left[\int_{s}^{t} \partial_{x} A e^{-r A} \partial_{x} a d r\right]_{h^{\gamma}} \leq \varepsilon^{\frac{1+\gamma}{2}} \int_{s}^{t}\left\|\partial_{x} A e^{-r A}\right\|_{\mathcal{L}\left(L^{\infty}(\mathbf{R}), h \gamma(\mathbf{R})\right)} \cdot\left|\partial_{x} a\right|_{\infty} d r \\
& \lesssim \varepsilon^{\frac{1+\gamma}{2}} \int_{s}^{t} r^{-1-\frac{1}{4}-\frac{\gamma}{4}} d r \cdot\|a\|_{B U C^{1}(\mathbf{R})} \\
& \lesssim \frac{4}{1+\gamma} \varepsilon^{\frac{1+\gamma}{2}} \frac{(t-s)^{\frac{1+\gamma}{4}}}{s^{\frac{1+\gamma}{4}} t^{\frac{1+\gamma}{4}}}\|a\|_{B U C^{1}(\mathbf{R})} \leq \frac{4}{1+\gamma}(t-s)^{\frac{1+\gamma}{4}}\|a\|_{B U C^{1}(\mathbf{R})} .
\end{aligned}
$$

Now we estimate $J_{2}$ by

$$
J_{2} \leq \int_{s}^{t}\left\|\partial_{x}^{3} e^{-(t-\tau) A}\right\|_{\mathcal{L}\left(h^{\gamma}(\mathbf{R}), h^{\gamma}(\mathcal{R})\right)} \cdot[\Xi(\tau)]_{h \gamma} d \tau \lesssim c_{1}\left(M, M_{\infty}\right) \int_{s}^{t}(t-\tau)^{-3 / 4} \tau^{\alpha-1} d \tau
$$

If $s<t / 2$, then we have

$$
\varepsilon^{\frac{1+\gamma}{2}} \int_{s}^{t}(t-\tau)^{-3 / 4} \tau^{\alpha-1} d \tau=\varepsilon^{\frac{1+\gamma}{2}}\left(\int_{s}^{t / 2}+\int_{t / 2}^{t}\right)(t-\tau)^{-3 / 4} \tau^{\alpha-1} d \tau
$$

For $\tau \in[s, t / 2]$, note that $\varepsilon \leq \tau$ and $\tau \leq t-\tau$. Thus, we observe

$$
\begin{aligned}
\varepsilon^{\frac{1+\gamma}{2}} \int_{s}^{t / 2}(t-\tau)^{-3 / 4} \tau^{\alpha-1} d \tau & \leq \varepsilon^{\frac{1+\gamma}{2}} \int_{s}^{t / 2}(t-\tau)^{-3 / 4+\gamma / 4}(t-\tau)^{-\gamma / 4} \tau^{\frac{1+\gamma}{2}} \tau^{\alpha-1} d \tau \\
& =\int_{s}^{t / 2}(t-\tau)^{-3 / 4+\gamma / 4}\left(\frac{\tau}{t-\tau}\right)^{\gamma / 4} d \tau \\
& \leq \int_{s}^{t / 2}(t-\tau)^{-3 / 4+\gamma / 4} d \tau \leq \frac{4}{1+\gamma}(t-s)^{\frac{1+\gamma}{4}}
\end{aligned}
$$

For $\tau \in[t / 2, t]$, we observe

$$
\begin{aligned}
\varepsilon^{\frac{1+\gamma}{2}} \int_{t / 2}^{t}(t-\tau)^{-3 / 4} \tau^{\alpha-1} d \tau & \leq \varepsilon^{\frac{1+\gamma}{2}}\left(\frac{t}{2}\right)^{\alpha-1} \int_{t / 2}^{t}(t-\tau)^{-3 / 4} d \tau \\
& =4 \varepsilon^{\frac{1+\gamma}{2}}\left(\frac{t}{2}\right)^{\alpha-1}\left(\frac{t}{2}\right)^{1 / 4} \\
& \leq 4 \cdot 2^{\frac{1+\gamma}{2}}\left(\frac{t}{2}\right)^{\frac{1+\gamma}{2}}\left(\frac{t}{2}\right)^{\alpha-3 / 4} \leq 2^{\frac{5+\gamma}{2}}(t-s)^{\frac{1+\gamma}{4}}
\end{aligned}
$$


If $s \geq t / 2$, then we calculate in the same way as (3.28),

$$
\begin{aligned}
\varepsilon^{\frac{1+\gamma}{2}} \int_{s}^{t}(t-\tau)^{-3 / 4} \tau^{\alpha-1} d \tau & \leq \varepsilon^{\frac{1+\gamma}{2}} \int_{t / 2}^{t}(t-\tau)^{-3 / 4} \tau^{\alpha-1} d \tau \\
& \leq 2^{\frac{5+\gamma}{2}}(t-s)^{\frac{1+\gamma}{4}}
\end{aligned}
$$

From (3.26)-(3.29) we have

$$
J_{2} \lesssim c_{1}(t-s)^{\frac{1+\gamma}{4}}
$$

$J_{3}$ is estimated by

$$
\begin{aligned}
J_{3} & \leq \varepsilon^{\frac{1+\gamma}{2}} \int_{0}^{s} \int_{s-\tau}^{t-\tau}\left\|\partial_{x}^{3} A e^{-r A}\right\|_{\mathcal{L}\left(h^{\gamma}(\mathbf{R}), h^{\gamma}(\mathbf{R})\right)}[\Xi(\tau)]_{h^{\gamma}} d \tau \\
& \lesssim c_{1} \varepsilon^{\frac{1+\gamma}{2}} \int_{0}^{s} \int_{s-\tau}^{t-\tau} r^{-1-3 / 4} \tau^{\alpha-1} d r d \tau \\
& =c_{1} \varepsilon^{\frac{1+\gamma}{2}} \int_{0}^{s} \int_{s-\tau}^{t-\tau} r^{-3 / 4+\gamma / 4} \cdot r^{-1-\gamma / 4} \tau^{\alpha-1} d r d \tau \\
& \leq c_{1} \varepsilon^{\frac{1+\gamma}{2}} \int_{0}^{s}(s-\tau)^{-1-\gamma / 4} \tau^{\alpha-1} d \tau \int_{s-\tau}^{t-\tau} r^{-3 / 4+\gamma / 4} d r \\
& \leq \frac{4}{1+\gamma} c_{1} \varepsilon^{\frac{1+\gamma}{2}}\left(\int_{0}^{s}(s-\tau)^{-1-\gamma / 4} \tau^{\alpha-1} d \tau\right)(t-s)^{\frac{1+\gamma}{4}} \\
& \leq \frac{4}{1+\gamma} c_{1}\left(\int_{0}^{1}(1-\sigma)^{-1-\gamma / 4} \sigma^{\alpha-1} d \sigma\right)(t-s)^{\frac{1+\gamma}{4}}
\end{aligned}
$$

From (3.25), (3.30) and (3.31) we conclude that $u \in C_{\frac{1+\gamma}{2}}^{\frac{1+\gamma}{4}}\left(J, h_{\text {even }}^{2+\gamma}(\mathbf{R})\right)$ and there exists a constant $C:=C\left(M, M_{\infty}\right)$ such that

$$
[u]_{C_{\frac{1+\gamma}{2}}^{\frac{1+\gamma}{4}}\left(J, h_{\text {even }}^{2+\gamma}(\mathbf{R})\right)} \leq C
$$

Proof of Theorem 3.4. From Lemma 3.5, $u_{\lambda}$ is uniformly bounded and equi-continuous. From AscoliArzela theorem, there exists a subsequence $\left\{u_{\lambda_{j}}\right\}_{j=1}^{\infty}$ such that $u_{\lambda_{j}}$ uniformly converges to $\tilde{u}$ on any compact sets in $L^{\infty}((0, T] \times \mathbf{R})$ up to the second order derivatives. Note that

$$
\begin{aligned}
\left\|a_{\lambda_{j}}\right\|_{B U C^{1}(\mathbf{R})} & =\left|a_{\lambda_{j}}\right|_{\infty}+\left|\partial_{x} a_{\lambda_{j}}\right|_{\infty} \\
& =\frac{1}{\lambda_{j}} \sup _{x}\left|a\left(\lambda_{j} x\right)\right|+\sup _{x}\left|a^{\prime}\left(\lambda_{j} x\right)\right| \\
& \leq|a|_{\infty}+\left|a^{\prime}\right|_{\infty}=\|a\|_{B U C^{1}(\mathbf{R}) .}
\end{aligned}
$$

From our method of constructing the solution, we observe that there exists $C^{\prime}:=C^{\prime}\left(M, M_{\infty}\right)$ such that

$$
\left\|u_{\lambda_{j}}\right\|_{B U C_{1-\alpha}\left(J, h_{\mathrm{even}}^{3+\gamma}(\mathbf{R})\right)} \lesssim C^{\prime}\left\|a_{\lambda_{j}}\right\|_{B U C^{1}(\mathbf{R})} \leq C^{\prime}\|a\|_{B U C^{1}(\mathbf{R})}
$$


This boundedness enables us to conclude that the third order derivative $\partial_{x}^{2} u_{\lambda_{j}}$ converges to $\partial_{x}^{3} \tilde{u}$ in $L^{\infty} *$-weak sense. Recall that

$$
\Xi_{\lambda_{j}}:=\left(\frac{1}{\left(1+\left(u_{\lambda_{j}}+U^{L}\right)_{x}^{2}\right)^{2}}-1\right) \partial_{x}^{3} u_{\lambda_{j}}-\frac{3\left(u_{\lambda_{j}}+U^{L}\right)_{x}\left(u_{\lambda_{j}}+U^{L}\right)_{x x}^{2}}{\left(1+\left(u_{\lambda_{j}}+U^{L}\right)_{x}^{2}\right)^{3}}
$$

The first term of (3.32) is the product of uniform convergence sequence and $L^{\infty} *$-weakly convergence sequence. The second term is a uniform convergence sequence. Thus, $\Xi\left(\left(u_{\lambda_{j}}+U^{L}\right)_{x},\left(u_{\lambda_{j}}+\right.\right.$ $\left.\left.U^{L}\right)_{x x},\left(u_{\lambda_{j}}+U^{L}\right)_{x x x}\right)$ converges to $\Xi\left(\left(\tilde{u}+U^{L}\right)_{x},\left(\tilde{u}+U^{L}\right)_{x x},\left(\tilde{u}+U^{L}\right)_{x x x}\right)$ as $\lambda_{j} \rightarrow \infty$ in $L^{\infty}$ *-weak sense. Thus

$$
\begin{aligned}
u_{\lambda_{j}} & =e^{-t A} a_{\lambda_{j}}-\int_{0}^{t} e^{-(t-s) A} \partial_{x} \Xi\left(\left(u_{\lambda_{j}}+U^{L}\right)_{x},\left(u_{\lambda_{j}}+U^{L}\right)_{x x},\left(u_{\lambda_{j}}+U^{L}\right)_{x x x}\right)(\tau) d \tau \\
& =\frac{1}{\lambda_{j}} e^{-t A} a\left(\lambda_{j} x\right)-\int_{0}^{t} e^{-(t-s) A} \partial_{x} \Xi\left(\left(u_{\lambda_{j}}+U^{L}\right)_{x},\left(u_{\lambda_{j}}+U^{L}\right)_{x x},\left(u_{\lambda_{j}}+U^{L}\right)_{x x x}\right)(\tau) d \tau \\
& \rightarrow \tilde{u}=0-\int_{0}^{t} e^{-(t-s) A} \partial_{x} \Xi\left(\left(\tilde{u}+U^{L}\right)_{x},\left(\tilde{u}+U^{L}\right)_{x x},\left(\tilde{u}+U^{L}\right)_{x x x}\right)(\tau) d \tau \quad \text { as } \lambda_{j} \rightarrow \infty
\end{aligned}
$$

$\tilde{u}$ is the mild solution with zero initial data, that is, $\tilde{u}$ is self-similar.

\section{A Appendix}

We show the key estimate of the technique developed by Angenent [1, Theorem 2.14] which is the extension of Da Prato-Grisvard construction [8]. The point is the estimate for the integrand which shall be stated in Lemma A.1 as follows.

Lemma A.1. Suppose that $0<\alpha \leq 1$ and $0<\theta<1$. Then there exists a constant $C>0$ such that

$$
r^{1-\theta}(1 \wedge t)^{1-\alpha} \int_{0}^{t}(r+t-s)^{\theta-2}(1 \wedge s)^{-1+\alpha} d s \leq C
$$

for $r, t \in \mathbf{R}^{+}$.

Proof. If $t \leq 2$,

$$
\begin{aligned}
I_{1}(r, t) & :=\int_{0}^{t / 2}(r+t-s)^{\theta-2}(1 \wedge s)^{-1+\alpha} d s=\int_{0}^{t / 2}(r+t-s)^{\theta-2} s^{-1+\alpha} d s \\
& \leq(r+t)^{\theta-2+\alpha} \int_{0}^{1 / 2}(1-\sigma)^{\theta-2} \sigma^{-\mu} d \sigma \leq c(r+t)^{\theta-2+\alpha}
\end{aligned}
$$

If $t \geq 2$,

$$
\begin{aligned}
I_{1}(r, t) & :=\int_{0}^{1}(r+t-s)^{\theta-2} s^{-1+\alpha} d s+\int_{1}^{t / 2}(r+t-s)^{\theta-2} d s \\
& \leq c\left\{(r+t)^{\theta-2+\alpha}+(r+t / 2)^{\theta-1}\right\} \leq c(r+1)^{\theta-1}
\end{aligned}
$$


Similarly, if $t \geq 2$,

$$
I_{2}(r, t):=\int_{t / 2}^{t}(r+t-s)^{\theta-2}(1 \wedge s)^{-1+\alpha} d s=\int_{t / 2}^{t}(r+t-s)^{\theta-2} d s \leq c r^{\theta-1} .
$$

If $t \leq 1$,

$$
I_{2}(r, t)=\int_{t / 2}^{t}(r+t-s)^{\theta-2} s^{-1+\alpha} d s \leq c t^{-1+\alpha} r^{\theta-1} .
$$

Lastly, if $1<t<2$,

$$
I_{2}(r, t)=\int_{t / 2}^{1}(r+t-s)^{\theta-2} s^{-1+\alpha} d s+\int_{1}^{t}(r+t-s)^{\theta-2} d s \leq c r^{\theta-1} .
$$

Now the assertion is obvious.

\section{Acknowledgments}

The authors are grateful to Professor Yoshihito Kohsaka for bringing the paper of Martin [20] to their attention. The work of the second author is partly supported by the Japan Society for the Promotion of Science through grant Kiban (S) 21224001, Kiban (A) 23244015, Houga 25610025. Most of work was done while the first author was a graduate student at the Mathematics Department of the University of Tokyo.

\section{References}

[1] Angenent, S. B. Nonlinear analytic semiflows. Proc. Roy. Soc. Edinburgh Sect A 115 (1990), 91-107.

[2] ASAI, T. On smoothing effect for higher order curvature flow equations. Adv. Math. Sci. Appl. 20 (2010), 483-509.

[3] ASAI, T. Quasilinear parabolic equation and its applications to fourth order equations with rough initial data. J. Math. Sci. Univ. Tokyo. 19 (2012), 507-532.

[4] ASAI, T. Analytic semigroup approach to higher order quasilinear parabolic problems. $\mathrm{PhD}$ thesis, The University of Tokyo (2013).

[5] Broadbridge, P., \& Tritscher, P. An integrable fourth-order nonlinear evolution equation applied to thermal grooving of metal surfaces. IMA J. Appl. Math. 53 (1994), 249-265.

[6] Cazenave, T., \& Weissler, F. B. Asymptotically self-similar global solutions of the nonlinear Schrödinger and heat equations. Math. Z. 228 (1998), 83-120. 
[7] Clément, Ph., \& Simonett, G. Maximal regularity in continuous interpolation spaces and quasilinear parabolic equations. J. Evol. Equ. 1 (2001), 39-67.

[8] Da Prato, G., \& Grisvard, P. Equations d'évolution abstraites nonlinéaires de type parabolique. Ann. Mat. Pura Appl. (4) 120 (1979), 329-396.

[9] Escher, J., MAyer, U. F., \& Simonett, G. The surface diffusion flow for immersed hypersurfaces. SIAM J. Math. Anal. 29 (1998), 1419-1433.

[10] Escher, J., \& MuChA, P. The surface diffusion flow on rough phase spaces. Discrete Contin. Dyn. Syst. 26 (2010), 431-453.

[11] Garcke, H., Iто, K., \& KohsaKa, Y. Linearized stability analysis of stationary solutions for surface diffusion with boundary conditions. SIAM J. Math. Anal. 36 (2005), 1031-1056.

[12] GARCKe, H., ITO, K., \& KohSAKA, Y. Nonlinear stability of stationary solutions for surface diffusion with boundary conditions. SIAM J. Math. Anal. 40 (2008), 491-515.

[13] Garcke, H., Ito, K., \& Kohsaka, Y. Surface diffusion with triple junctions: A stability criterion for stationary solutions. Adv. Differential Equations. 15 (2010), 437-472.

[14] Giga, Y., \& MiYaKawA, T. Navier-Stokes flow in $\mathbf{R}^{3}$ with measures as initial vorticity and Morrey spaces. Comm. Partial Differential Equations 14 (1989), 577-618.

[15] Hamamuki, N. Asymptotically self-similar solutions to curvature flow equations with prescribed contact angle and their applications to groove profiles due to evaporation-condensation. preprint.

[16] Iто, K., \& KohsakA, Y. Three phase boundary motion by surface diffusion: Stability of a mirror symmetric stationary solution. Interfaces and Free Boundaries. 3 (2001) 45-80.

[17] Iто, K., \& KohsaKa, Y. Three phase boundary motion by surface diffusion in triangular domain. Adv. Math. Sci. Appl. 11 (2001), 753-779.

[18] Kanel, J., Novick-Cohen, A., \& Vilenkin, A. Coupled surface and grain boundary motion: a travelling wave solution. Nonlinear Anal. 59 (2004), 1267-1292.

[19] LunARdi, A. Analytic Semigroups and Optimal Regularity in Parabolic Problems. Birkhäuser, Basel, (1995).

[20] Martin, P. A. Thermal grooving surface diffusion: Mullins revisited and extended to multiple grooves. Quart. Appl. Math. 67 (2009), 125-136.

[21] Mullins, W. W. Theory of thermal grooving. J. Appl. Phys. 28 (1957), 333-339. 
[22] ZHU, P. Asymptotic stability of the stationary solution to an initial boundary value problem for the Mullins equation of fourth order. J. Math. Phys. 50 (2009), 102707, 12 pp. 